Reflexiones y aportes para la formación de investigadores en Periodismo deportivo

Karen Alustiza

http://perio.unlp.edu.ar/ojs/index.php/question/article/view/4708

Cita sugerida: Alustiza, K. (2018). Reflexiones y aportes para la formación de investigadores en Periodismo deportivo.

Question, 1(59), e088. doi:https://doi.org/10.24215/16696581e088

\title{
Reflexiones y aportes para la formación de investigadores en Periodismo deportivo
}

\section{Reflections and contributions for the training of researchers in}

Sports journalism

Karen Alustiza kalustiza.adn@gmail.com

http://orcid.org/0000-0002-1063-4002

Facultad de Periodismo y Comunicación Social;

Universidad Nacional de La Plata (Argentina)

\section{Resumen}

El presente artículo es una reseña del Cuaderno de cátedra Técnicas de investigación social, producido por los docentes del espacio curricular "Técnicas de investigación social" de la Facultad de Periodismo y Comunicación Social de la Universidad Nacional de La Plata (FPyCS, UNLP). La obra se divide en tres partes, trazando un recorrido que se inicia con el surgimiento de las ciencias sociales y culmina abordando las particularidades de la investigación social. Al finalizar, se comparte una breve reflexión sobre los aportes de la obra en la formación de los periodistas deportivos y los comunicadores sociales.

Palabras clave: ciencia; Investigación social; metodología cualitativa; técnicas de investigación; deporte. 


\section{Abstract}

The present article is a review of the book of the seminar named Techniques of Social investigation, which was produced by the teachers of the 'Techniques of Social Investigation' curricular area in the Facultad de Periodismo y Comunicacion Social at the Universidad Nacional de La Plata (FPyCS,UNLP). The work is divided into three parts, tracing an outline that starts with the appearance of the social sciences and finishes by dealing with the distinctive features of the social investigation. By the end, it is shared a brief reflection on the contribution of the work to the training of sport journalists and social communicators.

Keywords: science; social investigation; qualitative methodology; research techniques; sports.

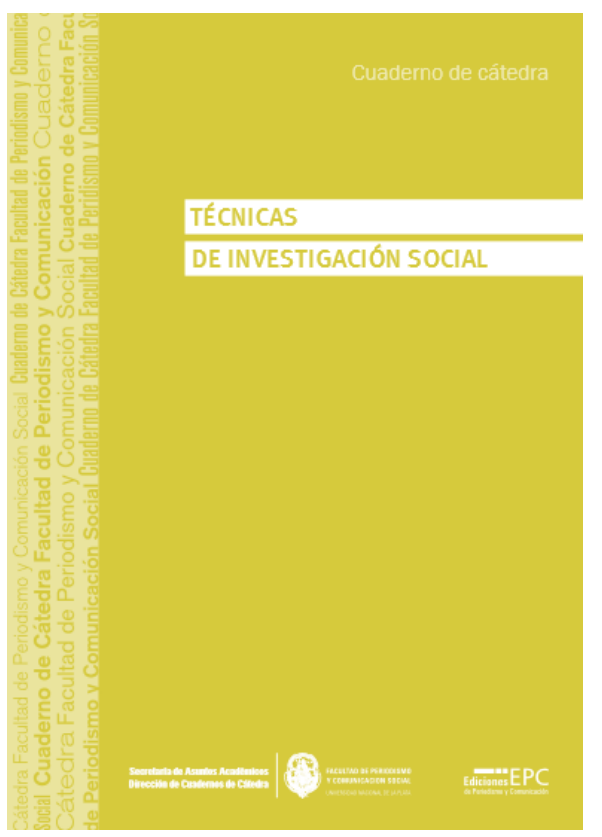

La obra es un e-book de acceso gratuito (1), publicado en 2017 por la Editorial de Periodismo y Comunicación (EPC). Constituye una guía de enseñanza de lectura ágil y sencilla, con el fin de que el lector pueda no solamente analizar los saberes producidos por otros sino también para entender cómo se gesta nuevo conocimiento científico. Reúne tres partes que ponen el foco en las ciencias sociales, en el sujeto investigador, en el objeto de estudio, en el trabajo de campo y en todas las decisiones que implican llevar adelante el proceso de conocer. Es una propuesta con un claro sentido pedagógico, cuyo abordaje teórico es fundamentado y sostenido con ejemplos concretos del campo deportivo. También se incluyen ejercicios, preguntas y propuestas de evaluación para los lectores, similares a las que desde hace tiempo se desarrollan en el marco de la cursada. Cabe resaltar que los temas de las mismas refieren a prácticas ligadas al universo deportivo.

El libro coordinado por Lucrecia Ametrano, por entonces profesora titular del espacio curricular "Técnicas de Investigación Social" de la Facultad de Periodismo de la Universidad Nacional de La Plata (FPyCS, UNLP), fue realizado por los siguientes autores: Pamela Vestfrid, licenciada y profesora en Comunicación social; Adrián Bonaparte, docente y licenciado en Ciencias 
antropológicas; Ramiro Adrado, licenciado y profesor en Comunicación social; y Francisco Huarte, Técnico Superior en Periodismo deportivo y Licenciado en Comunicación social. "Si podemos determinar una especificidad en el hombre, sería su necesidad constante de comprender y explicar el mundo que lo rodea" (Ametrano, 2017: 11). Esta afirmación es sostenida y argumentada en cada uno de los textos que componen la obra. Evidentemente, enseñar a investigar es una tarea muy compleja que universidades e instituciones educativas han decidido emprender con el propósito de impulsar la producción de conocimiento científico desde las ciencias sociales.

Una buena pregunta que nos introduce en el primer apartado es ¿Por qué conocemos? Como señala la autora, la producción de saberes es una actividad humana cuyos métodos y herramientas se han modificado a lo largo de la historia. El paso a la Edad Moderna transformó de manera significativa la vida económica, política, social y cultural; a su vez, las visiones del mundo con tintes religiosos dieron paso a nuevas formas de interpretación. Se produjo así una ruptura en los modos de pensar y explicar una realidad cada vez más compleja, y los hechos sociales que no podían abordarse con las técnicas que emplean las ciencias naturales, se constituyeron en el objeto de estudio de las ciencias sociales.

En ese sentido, Ametrano establece una relación directa entre sociedad y ciencia, situando el surgimiento de las ciencias sociales en los profundos cambios que experimentaron las sociedades europeas en los últimos siglos, a partir de acontecimientos históricos como la ruptura del antiguo régimen, la Revolución Francesa y la Revolución Industrial. En resumen, la primera parte del libro trata a grandes rasgos el origen y la caracterización de las ciencias sociales, además de presentar una sistematización de los modelos cuantitativo y cualitativo, y la definición de conceptos claves como "obstáculos epistemológicos", "ruptura epistemológica" y "vigilancia epistemológica". Con este propósito se retoman autores clásicos como Rosana Guber, Pierre Bourdieu, Esther Díaz, Émile Durkheim, entre otros.

Sin dudas, la generación de saberes es un reto difícil, incluso para quienes desde hace años están insertos en el campo de la investigación social. En la segunda parte titulada El desafío de producir conocimiento científico, Pamela Vestfrid describe la relación sujeto cognoscente/objeto de estudio y explica los obstáculos, tensiones y situaciones implicados en el proceso de conocer. Proceso, proyecto y diseño son los conceptos centrales desarrollados en este apartado. Además, se presentan las nociones básicas acerca de la elaboración de un proyecto de investigación (propuesta del trabajo final de la materia), con ejercicios y ejemplos provenientes del campo deportivo. 
En la tercera parte se desarrolla en profundidad la metodología cualitativa. El énfasis está puesto en el papel del sujeto investigador y los diferentes aspectos del trabajo de campo en ciencias sociales. Adrián Bonaparte reflexiona en torno a las múltiples decisiones involucradas en el proceso de investigación y describe algunas técnicas de recopilación de información, tales como la encuesta y cédula, la entrevista y la observación con y sin participación.

Francisco Huarte profundiza en la entrevista cualitativa y traza un paralelismo con la entrevista periodística para dar cuenta de las similitudes y diferencias entre la labor específica de periodistas e investigadores sociales. A continuación, Ramiro Adaro relata en primera persona su experiencia como adscripto de la cátedra de "Técnicas de Investigación Social" de la Tecnicatura en Periodismo deportivo. Pone de manifiesto las situaciones y dificultades a las que se enfrentan en el trabajo áulico.

En los anexos se presenta la propuesta del trabajo final de la materia (consiste en la elaboración de un proyecto de investigación con temáticas deportivas) y un relato acerca de una experiencia en investigación desarrollada por Ametrano. Es sumamente importante que la enseñanza se base no solamente en libros de metodología sino también que los docentes compartan en sus clases sus experiencias investigativas y los alumnos asuman un rol activo en la producción de conocimiento. Así es posible eliminar la división entre lo que se transmite en el aula y las decisiones metodológicas que se asumen en prácticas concretas. Además, es un impulso para que los estudiantes se animen a participar activamente en propuestas de investigación.

Una de las características fundamentales que comparten los textos de todos los autores es que poseen un vocabulario preciso y accesible, tanto para sus destinatarios directos (estudiantes de la Tecnicatura en Periodismo deportivo) como para lectores secundarios. Si bien la obra fue escrita y pensada fundamentalmente para los alumnos de la cátedra, es una fuente de consulta interesante para docentes, estudiantes y especialistas que, insertos en múltiples espacios o instituciones, llevan adelante tareas relacionadas con la investigación social y la producción de conocimiento. El desarrollo es ordenado y coherente: parte de la generalidad de la ciencia como tipo particular de conocimiento para finalizar con las técnicas aplicadas en el trabajo de campo por el sujeto investigador.

El libro es un gran aporte para la formación de los estudiantes por varias razones. En primer lugar, nos llama a considerar la importancia que actualmente tiene el deporte como objeto de estudio y, en ese sentido, se constituye en una guía teórica-práctica para la investigación de uno de los fenómenos sociales más influyentes de la cultura popular; en esa misma línea, advierte la necesidad de formar nuevos investigadores que asuman el desafío de seguir 
produciendo conocimiento en contextos cada vez más adversos; nos invita también a desnaturalizar ciertas prácticas del quehacer periodístico íntimamente relacionadas con la labor del investigador; por último, permite apreciar la complejidad de la generación de saberes desde las ciencias sociales llamándonos a afrontar el reto de continuar el camino emprendido por referentes como Roberto Da Matta y Eduardo Archetti, máximos exponentes de los estudios sobre deportes en América Latina.

Es necesario seguir reflexionando en torno a los modos en que se enseña a investigar en las universidades. Técnicas de investigación social es una guía valiosa por la claridad de los conceptos y los ejemplos concretos presentados por sus autores. Los desafíos para docentes, investigadores y jóvenes al momento de aventurarse a la producción de nuevos conocimientos son muchos. Afrontarlos con una buena guía escrita es el primer paso.

\section{Notas}

1 El libro puede descargarse gratuitamente del Repositorio de la Universidad Nacional de La Plata (SEDICI) o ingresando en el siguiente link https://bit.ly/2I2G0Xv

\section{Bibliografía}

Ametrano, L. (Coord.) (2017). Técnicas de Investigación Social. Cuaderno de cátedra (1ª ed.). La Plata, Argentina: EPC. Recuperado de https://bit.ly/2M3YUQg 